\title{
3 Research Square \\ Circulating markers of neutrophil extracellular traps (NETs) in patients with ovarian tumors
}

\author{
Arturas Dobilas ( $\square$ arturas.dobilas@med.lu.se ) \\ Lunds Universitet https://orcid.org/0000-0001-6884-2640 \\ Charlotte Thålin \\ Karolinska Institutet \\ Håkan Wallén \\ Karolinska Institutet \\ Christer Borgfeldt \\ Lunds Universitet
}

\section{Research article}

Keywords: Ovarian Cancer, tumor marker, NETs, cfDNA and H3Cit-DNA

Posted Date: July 28th, 2021

DOI: https://doi.org/10.21203/rs.3.rs-753660/v1

License: (a) (i) This work is licensed under a Creative Commons Attribution 4.0 International License. Read Full License

Version of Record: A version of this preprint was published at Anticancer Research on January 29th, 2022. See the published version at https://doi.org/10.21873/anticanres.15556. 


\section{Abstract \\ Background}

Inflammation is a hallmark of cancer, and emerging light is being shed on the neutrophil release of nuclear chromatin, referred to as neutrophil extracellular traps (NETs) in cancer and cancer associated thrombosis. The NET-specific marker citrullinated histone H3 (H3Cit) has been found to be elevated in plasma from patients with malignancies, suggesting the potential of NET markers, such as $\mathrm{H} 3 \mathrm{Cit}$, as novel cancer biomarkers.

\section{Objective}

To determine the levels of plasma H3Cit in blood in women with adnexal masses.

\section{Subjects and Method}

s: Peripheral blood samples were obtained preoperatively from 199 patients admitted for primary surgery of adnexal masses 2015-2017. Patients were grouped according to tumor type and stage of cancer. Plasma levels of H3Cit-DNA, cell free DNA (cfDNA) and the clinically implemented tumor marker cancer antigen 125 (CA125) were determined with ELISA.

\section{Results}

Plasma levels of H3Cit-DNA and cfDNA were not elevated in women with borderline or malignant ovarian tumors compared with women with benign tumors. Increased plasma levels of CA125 were detected in borderline and ovarian cancer stage I and stage IIIV compared with benign ovarian tumor patients $\left(p_{\text {trend }}<0.001\right.$ ). In Cox regression analysis high levels of Ca 125 dichotomized at $326 \mathrm{IU} / \mathrm{ml}$ (median) showed worse overall survival hazard ratio 1.9 (95\% C.I. 1.03-3.36; $p=0.038)$. No differences were found in the survival analyses in malignant ovarian tumors analyzing the cfDNA and H3Cit-DNA levels.

\section{Conclusion}

This study did not find any association nor prognostic association between the plasma levels of the NET marker H3Cit and ovarian cancer patients.

\section{Introduction}

Ovarian cancer (ovarian-/tubal-/peritoneal) is the sixth most frequent type of cancer and the fourth leading cause of cancer related death among Swedish women. Due to mild symptoms, two thirds of patients with ovarian cancer are diagnosed with advanced stage of the disease, and survival is higher when ovarian 
cancer is detected at an earlier stage (1). Recently, 5-year survival exceeded 50 percent in all stage of disease, reflecting more effective cytotoxic drugs and advances in surgical management.

Cancer antigen 125 (CA125), first identified in 1981, has been extensively studied for its utility in screening, early detection, prognosis, and monitoring of epithelial ovarian cancer (EOC) disease status (2), but its utility as a screening marker is limited due to poor sensitivity in early-stage ovarian cancer. Complex statistical algorithms along with a further understanding of the kinetics of biomarkers over time have improved the sensitivity and specificity of the biomarker assays (3).

Human epididymis protein 4 (HE4) is a novel tumor marker approved by the FDA for monitoring recurrence or progressive disease in patients with epithelial ovarian cancer (4). Overexpression of HE4 has been found in serous and endometrioid ovarian carcinomas. HE4 is included together with CA125 in the ROMA algorithm (risk of ovarian malignancy algorithm) (5) for preoperative assessment of suspected ovarian cancer. The biomarker panel suPAR(II-III), HE4, CA125 and age in premenopausal women improved discrimination of malignant and benign ovarian tumors (6) and high preoperative blood levels of HE4 have shown to predict poor prognosis in patients with ovarian cancer (7).

Inflammation plays a key role in tumorigenesis (8) and recent data emphasizes the contribution of neutrophils (9) and neutrophil release of nuclear chromatin, referred to as neutrophil extracellular traps (NETs) in cancer (10) and cancer-associated thrombosis (11). NETs were first discovered by Brinkman et al in 2004 (12) as a mechanism for trapping and killing bacteria by the innate immune system, but has recently been shown to play a central role in non-infectious conditions such as arterial and venous thrombosis $(13)$, autoimmunity and cancer $(8,9)$. Driven by the tumor environment, NETs have been implicated in tumor progression (14-16) by interacting with cancer cells and enhancing immune escape and angiogenesis, metastatic spread (17-20) by establishing a seeding bed for metastatic cells and promoting tumor cell migration, and in cancer-associated thrombosis $(21-24)$ by activation of platelets and coagulation factors as well as by providing a scaffold for platelets and red blood cells. The NETspecific marker citrullinated histone H3 (H3Cit) has been found elevated in plasma from patients with a variety of malignancies $(25,26)$ as well as from patients with cancer-associated thrombosis $(27,28)$, suggesting the potential of NET markers, such as H3Cit, as novel cancer biomarkers. NETs have also been shown to accumulate in peripheral blood vessels, compromising organ function in tumor-bearing mice (29) and markers of NETs have been detected in widespread microthrombi in cancer patients (27). Associations between high levels of circulating H3Cit and mortality in patients with advanced cancer has been reported (26). These data not only suggest that markers of NETs may be useful in cancer diagnostics and prognostics, but also that a NET-induced micro-thrombotic state may contribute to multi-organ failure and death in patients with advanced cancer, providing possible therapeutic targets.

The aim of this study was to analyze cell free DNA (cfDNA), a key component of NETs, and the NETspecific marker $\mathrm{H} 3 \mathrm{Cit}$ in plasma in patients with ovarian benign, borderline, and malignant tumors. The hypothesis was that there would be increased levels of NET markers in plasma in patients with malignant pelvic tumors. 


\section{Materials And Methods}

Patients. Peripheral blood samples were obtained preoperatively from 199 patients admitted for primary surgery of adnexal masses to the Department of Obstetrics and Gynecology in Lund, Sweden, October 2014-december 2017. Blood was collected in citrate tubes, and the plasma stored at $-80^{\circ} \mathrm{C}$ until analyzed. The 199 patients were chosen to get a representative material of ovarian tumor patients as well as some other pelvic malignancies from the biobank GUNNEL at Lund University, Medicon Village. All data were grouped according to tumor type and stage of cancer; Benign (B), Borderline (BOT), Stage I ovarian cancer (OvCa Stage I), Stage II-IV ovarian cancer (OvCa Stage II-IV) and Other cancer. dsDNA was quantified with Quant-iT PicoGreen dsDNA assay (Invitrogen) according to manufacturer's instructions. Nucleosomal H3Cit (H3Cit-DNA complexes) were quantified by an in-house ELISA as previously described (30).

CA125 was anlyzed in a commercial electrochemoluminescence immunoassay Elecsys CA125 kit $^{\text {TM }}$ (Roche Diagnostics Scandinavia AB, Bromma, Sweden) according to the manufacturer's instructions.

Statistical analyses

The Student's t-test, Pearson Chi-square test and ANOVA with Bonferroni's post hoc test were used in analyzing data. Trends across ordered groups were analyzed using linear regression with log-transformed values. Overall survival probabilities were calculated using the Kaplan-Meier method and the log-rank test. The Cox proportional hazard model was used in univariable survival and multivariable analyses. Point estimates are reported as hazard ratios (HR) and 95\% confidence intervals (Cl). Assumptions of proportional hazards were verified graphically where applicable. According to the power analysis with $a=$ $5 \%$ and power of $90 \%, 21$ patients were required in each group order to be able to find a $7 \%$ or more difference in mean values of the H3Cit variable between the groups. All comparisons were two-sided, and $5 \%$ significance level was used. Statistical analyses were performed using SPSSTM 26.02019 (IBM Corp, Armonk, NY, USA).

\section{Results}

Patients and data were grouped according to tumor type and stage of cancer as follows: Benign $(n=39)$, BOT $(n=32)$, Stage I $(n=23)$, stage II-IV $(n=99)$ and in other cancers $(n=6)$ (Table 1$)$. The youngest patients were in BOT group (mean age 53 years \pm 19.59 ) and the oldest patients were OvCa stage II-IV (mean 66 years \pm 9.38 ) (Table 1 ). The body mass index (BMI) of all groups of patients did not exceed 30 $\mathrm{kg} / \mathrm{m} 2$. The longest surgeries were in the OvCa Stage II-IV group $p<0.01)$ and the blood loss $(\mathrm{ml})$ were statistically larger in the OvCa Stage II-IV group than in the benign and borderline groups $(p<0.01)$. Of all the patients analyzed, the majority belonged to the ASA (American Society of Anesthesiologists) I-II groups (Table 1). Days to normal daily living was longer in cancer patients $(p=0.05)$. Among 98 patients responding to the 8 weeks follow-up questionnaire, no significant difference in thromboembolic events were confirmed between the OvCa Stage II-IV group (3 events out of 60 [5\%]) and the other patients with ovarian tumors (5 out of $33[15 \%]$ ). 
The dsDNA and H3Cit-DNA levels in plasma did not differ between patients with ovarian benign, borderline or malignant tumors or other cancer (Fig. 1a, 1b). The Ca125 levels in plasma were increased in BOT, OvCa stage I and OVCa stage II-IV compared with benign ovarian tumors and other cancer types in the pelvis $\left(p_{\text {trend }}<0.001\right)$.

In the survival analyses, benign and borderline tumors were excluded, and the biomarkers CA125, dsDNA and $\mathrm{H} 3 \mathrm{Cit}-\mathrm{DNA}$ analyzed as continuous variables as well as dichotomized and divided into quartiles. In the uni- and multivariable Cox regression analyses with continues variables increased levels of CA125 showed worse overall survival HR $2.0(95 \% \mathrm{Cl} 1.2-3.1, \mathrm{p}=0.004)$. No differences were found in the survival analyses in malignant ovarian tumors analyzing the dsDNA and H3Cit-DNA levels. In the univariable Cox regression analysis high levels of $\mathrm{Ca} 125$ dichotomized at $326 \mathrm{lU} / \mathrm{ml}$ (median) showed worse overall survival hazard ratio $1.9(95 \%$ C.I. $1.03-3.36 ; p=0.038)$ and log rank test $(p=0.034)$ (median follow-op time 37 months).

\section{Discussion}

Over the last decade, the interest in NETs in cancer progression has increased (31). Neutrophils are being increasingly recognized as an important element in tumor progression and have been shown to exert important effects at nearly every stage of tumor progression (32). Neutrophil response varies depending on stimuli (33). Stimuli in the tumour microenvironment result in activation of neutrophils with various antiand pro-tumour phenotypes (10). Markers of NET formation, such as H3Cit, have been found in plasma from cancer patients with acute microangiopathies $(27,34)$. In animal models, NET formation has been detected in the omentum of ovarian tumor-bearing mice before metastasis (35). It was also found that omental metastasis was decreased in mice with neutrophil-specific deficiency of PAD4 (peptidylarginine deiminase 4), an enzyme that is essential for NET formation (35). Based on these data, we hypothesized that circulating $\mathrm{H} 3 \mathrm{Cit}$ levels may be increased in ovarian cancer patients. Our results did not verify our hypothesis as the levels of H3Cit-DNA and cfDNA were not increased in patients with ovarian cancer and no associations were found between the levels of these plasma markers and ovarian cancer stage.

Cancer-associated NET formation likely depends on tumor type. NET formation has been associated with chronic autoimmune and autoinflammatory illnesses $(9,36,37)$ and implicated in the dissemination of cancer (10). In a murine model of chronic myelogenous leukemia the link between NET formation and cancer was demonstrated, isolated neutrophils were more prone to NET formation in vitro (22). In a study using mice bearing human pancreatic tumors, high levels of cfDNA and H3Cit in plasma correlated to spontaneous pulmonary thrombosis (38). In mouse models of breast cancer, non-small-cell lung carcinoma and chronic myeloid leukemia neutrophils indicated higher capacity to form and release NET $(10,32,39)$.

The CA125 ovarian cancer marker was in this study as expected higher in advanced stage III and IV cancer patients compared with borderline and early stage tumors (6). However, CA125 may be elevated in other benign gynecological diseases such as endometriosis and other medical conditions which reduce sensitivity and specificity of the biomarker CA125 (40). 
Many studies have shown the surgical impact on the survival for patients with ovarian cancer (41). To improve survival maximal cytoreductive tumor debulking surgery is advocated to reach no residual tumor $(42,43)$. Thorough tumor debulking often leads to longer operations, increasing the risk of venous thromboembolism (VTE). An important step is to classify patients into high and low risk of ovarian cancer so especially advanced ovarian cancer patients may be treated at tertiary surgical centers of excellence to reduce the risk of complications including VTE thus improving patient survival (44). In this study no increased incidence of VTE events were found in the OvCa group compared with the patients having surgery without the cancer diagnosis which may be due to the recommendations of prolonged anticoagulations prophylaxis in 30 days in ovarian cancer patients.

Elevations of plasma H3Cit in patients with advanced cancer patients compared with age-matched healthy individuals has earlier been reported (26). Several studies investigating cancer-associated NET formation (25) suggest that biomarkers of NET formation are associated with the occurrence of VTE in cancer patients (45). Patients with ovarian cancer are known to be at high risk for VTE $(46,47)$, but only VTE complicated by symptomatic pulmonary embolism have been identified to have a negative impact on survival (44).

This is, to our knowledge, the first study investigating plasma levels of cfDNA and H3Cit-DNA in patients with ovarian tumors. The Ca125 levels in the different ovarian tumor groups showed expected results in line with earlier studies indicating that the plasma analyzes in the patient groups behaved as expected. The number of patients in all ovarian tumor groups were according to the power analysis large enough. Some events of VTE may however have been missed since not all patients had complete follow-up.

\section{Conclusion}

This study did not find elevated levels of H3Cit or cfDNA in plasma from ovarian cancer patients. Further studies evaluating the role of NET formation in various cancer cohorts, especially those with known increased risk for thromboembolic events, are needed.

\section{Declarations}

\section{Acknowledgments}

We are grateful to all the women who participated in this study, to the staff at the Department of Obstetrics and Gynecology, Lund University Hospital, for assistance with collecting the

blood samples and to the laboratory staff at the Division of Oncology, Medicon Village, Lund University, for help with all practical issues concerning the preparation and shipping of

samples, and the laboratory staff at Clinical Research Center, Danderyd Hospital performing the NET analyses. 
Ethics: Written informed consent was obtained from all study participants. Ethical approval was granted by the Ethical Review Board at the Faculty of Medicine, Lund University, Sweden. Dnr 4952016 (amendment to Dnr 558-2004 and 94-2006).

\section{Author Contributions}

Conceptualization: Arturas Dobilas, Charlotte Thålin, Håkan Wallén, Christer Borgfeldt.

Data curation and formal statistical analyzes: Arturas Dobilas, Christer Borgfeldt

Investigation: Arturas Dobilas, Charlotte Thålin, Håkan Wallén, Christer Borgfeldt.

Visualization: Arturas Dobilas

Writing - original draft: Arturas Dobilas

Writing - review \& editing: Arturas Dobilas, Charlotte Thålin, Håkan Wallén, Christer Borgfeldt.

Funding: The study was supported by funds from the Swedish Cancer Foundation and Regional funds Region Skåne. The funders had no role in study design, data collection and analysis, decision to publish, or preparation of the manuscript.

Consent for publication: All authors agree to publish this article.

Competing interests: The authors declare no competing interests.

Availability of data and materials: All data are achieved according to the Swedish Act concerning the Ethical Review of Research Involving Humans to attain confidentiality and are available on reasonable request.

Competing interests: The authors declare that they have no conflicts of interest.

\section{References}

1. Äggstockscancer NVf. National Guidelines for Epithelial Ovarian Cancer 2019 [Available from: https://www.cancercentrum.se/syd/cancerdiagnoser/gynekologi/kvalitetsregister/statistik/aggstock/.

2. Bast RC Jr, Feeney M, Lazarus H, Nadler LM, Colvin RB, Knapp RC. Reactivity of a monoclonal antibody with human ovarian carcinoma. Journal of Clinical Investigation. 1981;68(5):1331-7.

3. Moore RG, MacLaughlan S, Bast RC Jr. Current state of biomarker development for clinical application in epithelial ovarian cancer. Gynecol Oncol. 2010;116(2):240-5.

4. Moore RG, McMeekin DS, Brown AK, DiSilvestro P, Miller MC, Allard WJ, et al. A novel multiple marker bioassay utilizing HE4 and CA125 for the prediction of ovarian cancer in patients with a pelvic mass. Gynecol Oncol. 2009;112(1):40-6. 
5. Moore RG, Jabre-Raughley M, Brown AK, Robison KM, Miller MC, Allard WJ, et al. Comparison of a novel multiple marker assay vs the Risk of Malignancy Index for the prediction of epithelial ovarian cancer in patients with a pelvic mass. Am J Obstet Gynecol. 2010;203(3):228.e1-6.

6. Leandersson P, Kalapotharakos G, Henic E, Borgfeldt H, Petzold M, Høyer-Hansen G, et al. A biomarker panel increases the diagnostic performance for epithelial ovarian cancer type i and II in young women. Anticancer Res. 2016;36(3):957-66.

7. Kalapotharakos G, Asciutto C, Henic E, Casslén B, Borgfeldt C. High preoperative blood levels of HE4 predicts poor prognosis in patients with ovarian cancer. Journal of Ovarian Research. 2012;5(1).

8. Grivennikov SI, Greten FR, Karin M. Immunity, inflammation, and cancer. Cell. 2010;140(6):883-99.

9. Brostjan C, Oehler R. The role of neutrophil death in chronic inflammation and cancer. Cell Death Discovery. 2020;6(1).

10. Wu L, Saxena S, Awaji M, Singh RK. Tumor-Associated Neutrophils in Cancer: Going Pro. Cancers (Basel). 2019;11(4):564.

11. Abdol Razak NB, Jones G, Bhandari M, Berndt MC, Metharom P. Cancer-Associated Thrombosis: An Overview of Mechanisms, Risk Factors, and Treatment. Cancers (Basel). 2018;10(10):380.

12. Brinkmann V, Reichard U, Goosmann C, Fauler B, Uhlemann Y, Weiss DS, et al. Neutrophil Extracellular Traps Kill Bacteria Science. 2004;303(5663):1532-5.

13. Thålin C, Hisada Y, Lundström S, Mackman N, Wallén H. Neutrophil Extracellular Traps: Villains and Targets in Arterial, Venous, and Cancer-Associated Thrombosis. Arterioscler Thromb Vasc Biol. 2019;39(9):1724-38.

14. Arelaki S, Arampatzioglou A, Kambas K, Papagoras C, Miltiades P, Angelidou I, et al. Gradient Infiltration of Neutrophil Extracellular Traps in Colon Cancer and Evidence for Their Involvement in Tumour Growth. PLoS One. 2016;11(5):e0154484.

15. Demers M, Wong SL, Martinod K, Gallant M, Cabral JE, Wang Y, et al. Priming of neutrophils toward NETosis promotes tumor growth. Oncoimmunology. 2016;5(5):e1134073.

16. Guglietta S, Chiavelli A, Zagato E, Krieg C, Gandini S, Ravenda PS, et al. Coagulation induced by C3aRdependent NETosis drives protumorigenic neutrophils during small intestinal tumorigenesis. Nature communications. 2016;7:11037.

17. Cools-Lartigue J, Spicer J, McDonald B, Gowing S, Chow S, Giannias B, et al. Neutrophil extracellular traps sequester circulating tumor cells and promote metastasis. J Clin Invest. 2013;123:3446-58.

18. Park J, Wysocki RW, Amoozgar Z, Maiorino L, Fein MR, Jorns J, et al. Cancer cells induce metastasissupporting neutrophil extracellular DNA traps. Science translational medicine. 2016;8(361):361ra138.

19. Tohme S, Yazdani HO, Al-Khafaji AB, Chidi AP, Loughran P, Mowen K, et al. Neutrophil Extracellular Traps Promote the Development and Progression of Liver Metastases after Surgical Stress. Cancer Res. 2016;76(6):1367-80.

20. Najmeh S, Cools-Lartigue J, Rayes RF, Gowing S, Vourtzoumis P, Bourdeau F, et al. Neutrophil Extracellular Traps sequester circulating tumor cells via beta1-integrin mediated interactions. Int J Cancer. 2017;140(10):2321-30. 
21. Guglietta S, Rescigno M. Hypercoagulation and complement: Connected players in tumor development and metastases. Semin Immunol. 2016.

22. Demers M, Krause DS, Schatzberg D, Martinod K, Voorhees JR, Fuchs TA, et al. Cancers predispose neutrophils to release extracellular DNA traps that contribute to cancer-associated thrombosis. Proc Natl Acad Sci U S A. 2012;109(32):13076-81.

23. Yang C, Sun W, Cui W, Li X, Yao J, Jia X, et al. Procoagulant role of neutrophil extracellular traps in patients with gastric cancer. Int J Clin Exp Pathol. 2015;8(11):14075-86.

24. Thalin C, Demers M, Blomgren B, Wong SL, von Arbin M, von Heijne A, et al. NETosis promotes cancerassociated arterial microthrombosis presenting as ischemic stroke with troponin elevation. Thrombosis research. 2016;139:56-64.

25. Grilz E, Mauracher LM, Posch F, Königsbrügge O, Zöchbauer-Müller S, Marosi C, et al. Citrullinated histone $\mathrm{H} 3$, a biomarker for neutrophil extracellular trap formation, predicts the risk of mortality in patients with cancer. Br J Haematol. 2019;186(2):311-20.

26. Thålin C, Lundström S, Seignez C, Daleskog M, Lundström A, Henriksson P, et al. Citrullinated histone $\mathrm{H} 3$ as a novel prognostic blood marker in patients with advanced cancer. PLoS One. 2018;13(1):e0191231-e.

27. Thålin $C$, Demers $M$, Blomgren $B$, Wong SL, von Arbin M, von Heijne A, et al. NETosis promotes cancerassociated arterial microthrombosis presenting as ischemic stroke with troponin elevation. Thromb Res. 2016;139:56-64.

28. Mauracher LM, Posch F, Martinod K, Grilz E, Däullary T, Hell L, et al. Citrullinated histone H3, a biomarker of neutrophil extracellular trap formation, predicts the risk of venous thromboembolism in cancer patients. J Thromb Haemost. 2018;16(3):508-18.

29. Cedervall J, Dimberg A, Olsson A-K. Tumor-induced neutrophil extracellular traps-drivers of systemic inflammation and vascular dysfunction. Oncoimmunology. 2015;5(3):e1098803-e.

30. Thålin C, Aguilera K, Hall NW, Marunde MR, Burg JM, Rosell A, et al. Quantification of citrullinated histones: Development of an improved assay to reliably quantify nucleosomal H3Cit in human plasma. J Thromb Haemost. 2020;18(10):2732-43.

31. Rivera-Franco MM, Leon-Rodriguez E, Torres-Ruiz JJ, Gómez-Martín D, Angles-Cano E de la. Luz Sevilla-González M. Neutrophil Extracellular Traps Associate with Clinical Stages in Breast Cancer. Pathology Oncology Research. 2020;26(3):1781-5.

32. Cools-Lartigue J, Spicer J, Najmeh S, Ferri L. Neutrophil extracellular traps in cancer progression. Cell Mol Life Sci. 2014;71(21):4179-94.

33. Scapini P, Lapinet-Vera JA, Gasperini S, Calzetti F, Bazzoni F, Cassatella MA. The neutrophil as a cellular source of chemokines. Immunol Rev. 2000;177:195-203.

34. Demers M, Wagner DD. NETosis: a new factor in tumor progression and cancer-associated thrombosis. Semin Thromb Hemost. 2014;40(3):277-83.

35. Lee W, Ko SY, Mohamed MS, Kenny HA, Lengyel E, Naora H. Neutrophils facilitate ovarian cancer premetastatic niche formation in the omentum. J Exp Med. 2019;216(1):176-94. 
36. Rosales C. Neutrophil. A Cell with Many Roles in Inflammation or Several Cell Types? Front Physiol. 2018;9:113-

37. Kaplan MJ. Role of neutrophils in systemic autoimmune diseases. Arthritis Res Ther. 2013;15(5):219-.

38. Hisada Y, Grover SP, Maqsood A, Houston R, Ay C, Noubouossie DF, et al. Neutrophils and neutrophil extracellular traps enhance venous thrombosis in mice bearing human pancreatic tumors. Haematologica. 2020;105(1):218-25.

39. Garley M, Jabłońska E, Dąbrowska D. NETs in cancer. Tumor Biology. 2016;37(11):14355-61.

40. Moore RG, McMeekin DS, Brown AK, DiSilvestro P, Miller MC, Allard WJ, et al. A novel multiple marker bioassay utilizing HE4 and CA125 for the prediction of ovarian cancer in patients with a pelvic mass. Gynecol Oncol. 2009;112(1):40-6.

41. Wimberger P, Lehmann N, Kimmig R, Burges A, Meier W, Du Bois A. Prognostic factors for complete debulking in advanced ovarian cancer and its impact on survival. An exploratory analysis of a prospectively randomized phase III study of the Arbeitsgemeinschaft Gynaekologische Onkologie Ovarian Cancer Study Group (AGO-OVAR). Gynecol Oncol. 2007;106(1):69-74.

42. Carney ME, Lancaster JM, Ford C, Tsodikov A, Wiggins CL. A population-based study of patterns of care for ovarian cancer: who is seen by a gynecologic oncologist and who is not? Gynecol Oncol. 2002;84(1):36-42.

43. Chang SJ, Hodeib M, Chang J, Bristow RE. Survival impact of complete cytoreduction to no gross residual disease for advanced-stage ovarian cancer: A meta-analysis. Gynecol Oncol. 2013;130(3):493-8.

44. Fotopoulou C, duBois A, Karavas AN, Trappe R, Aminossadati B, Schmalfeldt B, et al. Incidence of venous thromboembolism in patients with ovarian cancer undergoing platinum/paclitaxel-containing first-line chemotherapy: an exploratory analysis by the Arbeitsgemeinschaft Gynaekologische Onkologie Ovarian Cancer Study Group. J Clin Oncol. 2008;26(16):2683-9.

45. Mauracher LM, Posch F, Martinod K, Grilz E, Däullary T, Hell L, et al. Citrullinated histone H3, a biomarker of neutrophil extracellular trap formation, predicts the risk of venous thromboembolism in cancer patients. J Thromb Haemost. 2018;16(3):508-18.

46. Rodriguez AO, Wun T, Chew H, Zhou H, Harvey D, White RH. Venous thromboembolism in ovarian cancer. Gynecol Oncol. 2007;105(3):784-90.

47. Leandersson P, Granåsen G, Borgfeldt C. Ovarian Cancer Surgery - A Population-based Registry Study. Anticancer Res. 2017;37(4):1837-45.

\section{Tables}


Table 1. Patients characteristics

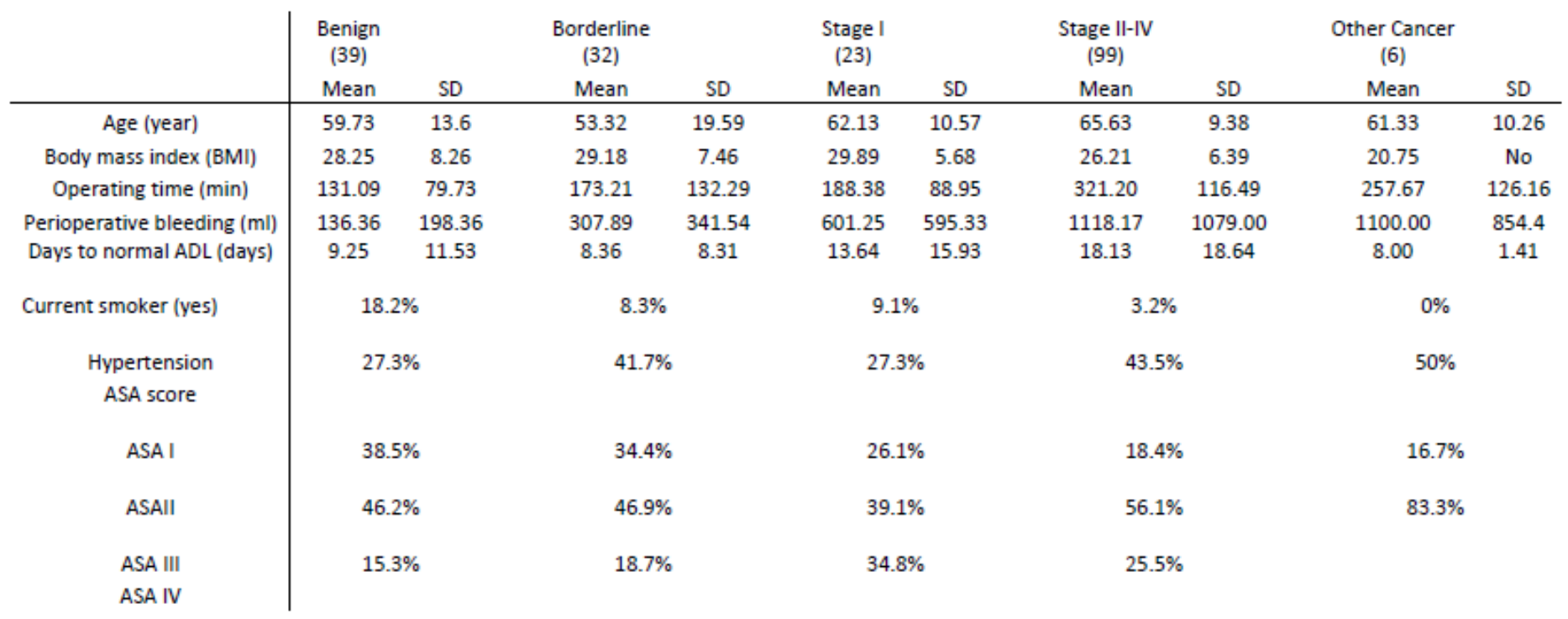

$\mathrm{ADL}=$ activity of daily living

ASA: The American Society of Anesthesiologists physical status classification system;

FIGO Stage: The International Federation of Gynecology and Obstetrics;

\section{Figures}

1a

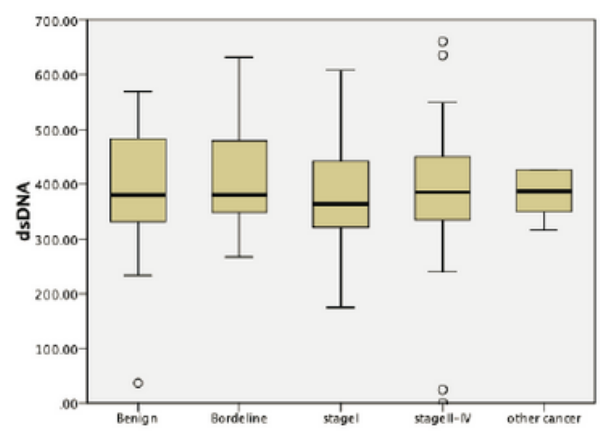

$1 \mathrm{~b}$

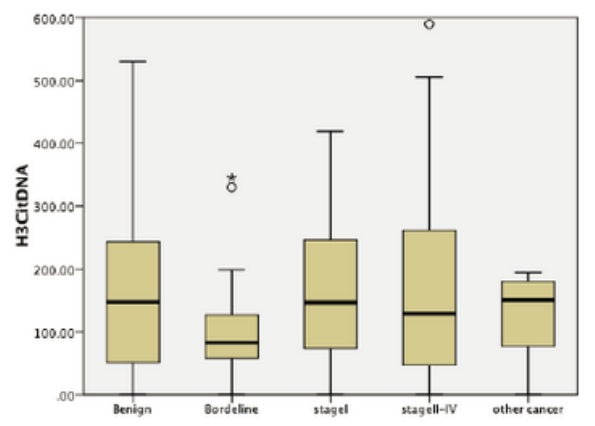

$1 c$

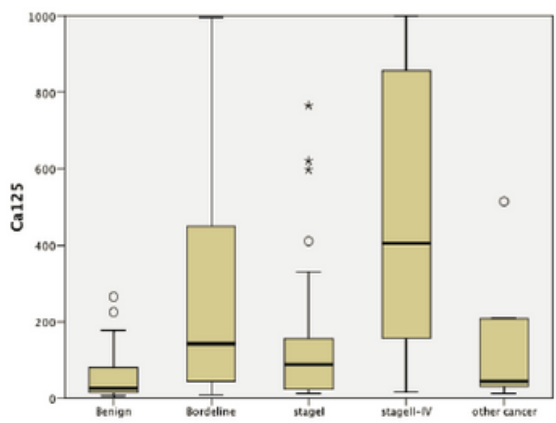

\section{Figure 1}

Figure 1 a Plasma levels of cfDNA did not differ between patients with ovarian benign, borderline or malignant tumors. Figure $1 \mathrm{~b}$ Plasma levels of H3Cit-DNA did not differ between patients with ovarian benign, borderline or malignant tumors. Figure $1 \mathrm{c} \mathrm{Ca} 125$ in plasma was increased in borderline, in stage I and stage II-IV ovarian cancer patients but not patients with other than ovarian cancer. 